\title{
Analisis espacial de la reforma fiscal en México en la LVIII Legislatura: un ejercicio aplicado de preferencias radiales y simétricas
}

Spatial analysis of tax reform in Mexico in LVIII Legislature:

an exercise applied preferences and symmetrical radial

\author{
Ignacio M. López Sandoval* "
}

\section{Resumen}

El objetivo de este trabajo es explicar a través de la teoría de jugadores de veto, como un ejercicio de aplicación de preferencias radiales y simétricas, porque no procedió la Reforma Fiscal en la LVIII legislatura. Esta aplicación empírica de la teoría de los jugadores de veto para el caso de México se inserta en el problema de división de poderes y las relaciones ejecutivolegislativo que ha impactado a las nuevas democracias Latinoamericanas.

El trabajo se divide en dos partes: la primera plantea los principales elementos teóricos del análisis espacial y de la teoría de los jugadores de veto. La segunda parte, se centra en la aplicación de la teoría deVeto Player (VP) en el análisis de los resultados de la Reforma Fiscal en la LVIII legislatura de la Cámara de Diputados.

Palabras clave:

- Jugadores de veto

- Preferencias

- Análisis espacial

- Reforma fiscal

\section{Abstract}

The aim of this work is explained by the theory of veto players, as an exercise in applying radial and symmetric preferences, because it did not carry the tax reform in the LVIII Legislature. This empirical application of the theory of veto players in the case of Mexico is inserted into the problem of separation of powers and executive-legislative relations that has impacted the new Latin American democracies.

The work is divided into two parts: the first presents the main theoretical elements of spatial analysis and the theory of veto players. The second part focuses on the application of the theory of Veto Player (VP) in the analysis of the results of the tax reform in the LVIII Legislature of the Chamber of Deputies.

Keywords:

- Veto player

- Preferences

- Spatial analysis

- Tax reform

JEL: C13, Q30, O13

\section{Introducción}

El año 2000 fue un punto de quiebre en el sistema político mexicano. Después de más de 60 años en el gobierno, el Partido Revolucionario Institucional (PRI) era derrotado por la Alianza para el Cambio (conformada por el Partido Acción Nacional y el Partido Verde Ecologista). Aun cuando las expectativas de los cambios económicos, políticos y sociales de la población y de ciertos sectores empresariales eran enormes en la nueva gestión del llamado Gobierno del Cambio, los resultados no fueron los esperados.

Si bien es cierto que el PAN controlaba el Poder Ejecutivo y tenía 35.9 y 42.4\% ${ }^{1}$ en la Cámara de Senadores y Diputados, respectivamente; el Poder Legislativo Federal en conjunto seguía siendo influido por las decisiones del

\footnotetext{
${ }^{1}$ Reynoso, D. (2004), Decisionismo y pluralismo en América Latina, Mimeo, p. 20.

* Profesor Investigador del Departamento de Estudios Institucionales de la Universidad Autónoma Metropolitana-Unidad Cuajimalpa.
} 
PRI, además de mantener más de 50\% de los Gobiernos y Congresos Locales. En este sentido el PRI pudo frenar los cambios sustantivos que el Gobierno del Presidente Vicente Fox había prometido a sus electores. Por lo menos uno de los asuntos más importantes en la agenda del nuevo Gobierno fue bloqueado: la Reforma Fiscal.

El objetivo de este trabajo es explicar a trasvés de la teoría de los jugadores de veto (Veto Players), porque no procedió la Reforma Fiscal en la LVIII legislatura de la Cámara de Diputados. Esta aplicación empírica de la teoría de los jugadores de veto para el caso de México se inserta en el problema de división de poderes y las relaciones ejecutivo-legislativo que ha impactado a las nuevas democracias Latinoamericanas.

El trabajo se divide en dos partes: la primera plantea los principales elementos teóricos del análisis espacial y de la teoría de los jugadores de veto que se utilizaran en la parte empírica, incorporando elementos adicionales de otras teorías que estudian a los grupos de presión, el intercambio de votos, o el voto estratégico, que podría modificar sustancialmente las preferencias de los jugadores.

Y la segunda parte, se centra en la aplicación de la teoría de Veto Player (vp) en el análisis de los resultados de la Reforma Fiscal en la LVIII legislatura de la Cámara de Diputados. Destacando como el cambio de preferencias del PRI, uno de los principales jugadores de veto, no permitió la modificación del Status Quo (sQ).

\section{Elementos teóricos del análisis espacial en la teoria de los Jugadores de Veto}

En este apartado se subrayan tres elementos teóricos importantes: a) el análisis espacial, realzando la importancia de las preferencias radiales y simétricas para obtener un votante mediano multidimensional; $b$ ) la teoría de los jugadores de veto (Veto Players) destacando los elementos más importantes de esta teoría y c) la teoría tradicional de la elección pública con relación al poder legislativo.

\section{a) Análisis espacial}

En cuanto al análisis espacial, tenemos que los primeros trabajos teóricos corresponden a Duncan Black (1948), que mediante el teorema del votante mediano 
Economía Informa núm. 389 noviembre - diciembre • 2014 | " " " " " " " " " " -

estableció la existencia de un ganador Condorcet. ${ }^{2}$ Este teorema fue generalizado posteriormente por Davis-Hinich-Plott-Sloss (1966, 1967, 1973) quienes establecieron un espacio multidimensional de temas, en el que un ganador Condorcet existe si y solo si un votante mediano multidimensional existe, lo cual requiere una clase muy especial de preferencias. ${ }^{3}$ Este tipo de preferencias suponen que los actores políticos (legisladores o votantes) tienen un punto ideal en cada asunto y que la utilidad de los actores disminuye cuando se alejan de esta política ideal. En el caso bidimensional ${ }^{4}$ estas preferencias radiales y simétricas, registran el conjunto de resultados o el espacio de política en dos dimensiones, estableciendo el dominio sobre el cual las preferencias se pueden expresar. Este tipo de análisis espacial y de preferencias son muy utilizadas por la teoría de los jugadores de veto. ${ }^{5}$

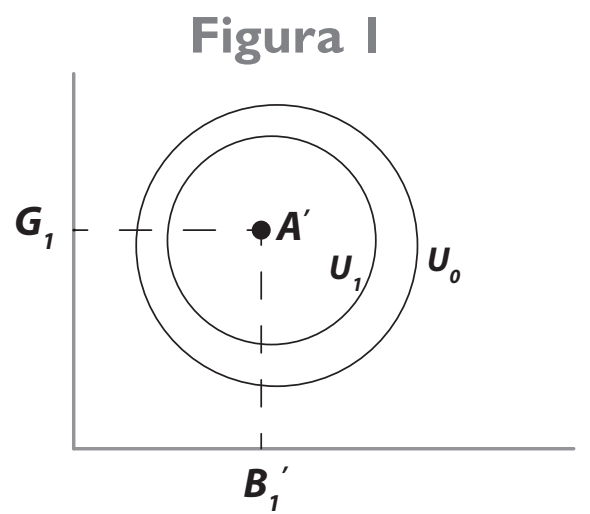

\footnotetext{
${ }^{2}$ Si los votantes tienen preferencias de un solo pico (single-peaked) ubicadas a lo largo de en una dimensión espacial y en un sólo asunto, la preferencia mediana es un ganador Condorcet. Algunos supuestos adicionales de este teorema son: a) número impar de votantes; $b$ ) participación completa; y c) voto sincero. Si se cumplen todos estos supuestos, decimos que un ganador Condorcet es aquel resultado que derrota a cada una de las otras propuestas en una votación por mayoría.

${ }^{3}$ Mueller, D. Perspectives on Public Choice. A. Handbook. Cambrige University Press, U.S.A 1997. pp. 248-249.

${ }^{4}$ En dos dimensiones las curves de indiferencia están centradas en el punto ideal del agente político, son curvas de indiferencia de punto de saciedad en forma de circulo. En más de dos dimensiones los contornos de indiferencia serán esferas o hiperesferas en más de cuatro dimensiones.

${ }^{5} \mathrm{Si}$ bien es cierto que las preferencias simétricas y radiales facilitan el análisis de la teoría de los jugadores de veto, las preferencias elípticas de punto de saciedad podrían ser mas realista; aunque modificarían algunos de los principales resultados de los jugadores individuales de veto. Tsebelis, G. (2002): Veto Players: How Political Institutions Work, Russell Sage Fundation, Princeton. Págs. 45-48.
} 
Las curvas $\boldsymbol{U}_{1}$ y $\boldsymbol{U}_{0}$ indican el nivel de utilidad de los agentes políticos, la cual aumenta cuando se acercan al punto $\boldsymbol{A}$ (Figura 1). La función de utilidad se maximiza en este punto, pero disminuye cuando aumenta la distancia al punto ideal $\boldsymbol{A} .^{6}$ Los puntos de los círculos con el radio más pequeño indica que nos acercamos más al punto preferido. ${ }^{7}$

Figura 2

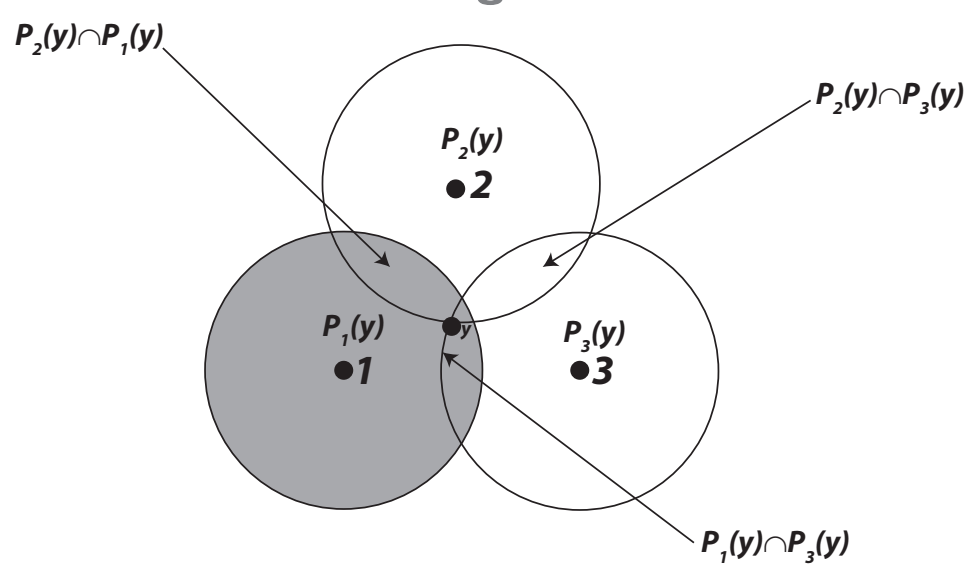

En el análisis espacial es importante ubicar al votante mediano, un primer acercamiento es delimitar las regiones de negociación marcadas por las intersecciones (Figura No 2): $\mathrm{P}_{1}(\mathrm{y}) \cap \mathrm{P}_{2}(\mathrm{y})$ que nos dan los puntos preferidos del agente 1 y 2 ; la intersección $\mathrm{P}_{2}(\mathrm{y}) \cap \mathrm{P}_{3}(\mathrm{y})$ determina los puntos preferidos de 2 y 3 y $\mathrm{P}_{1}(\mathrm{y}) \cap \mathrm{P}_{3}(\mathrm{y})$ los puntos preferidos de 1 y 3 , es decir, las posibles mayorías que se pueden formar con tres agentes. La unión de estos tres pétalos es el conjunto ganador de y o W(y). ${ }^{8}$ Cada intersección especifica una coalición de mayoría preferida a $\mathrm{y}^{9}$

\footnotetext{
${ }^{6}$ Todas las curvas de indiferencia son equidistantes a éste punto y cualquier punto sobre unas mismas curvas es igualmente preferido.

${ }^{7}$ Shepsle, K. A. y Bonchek, M. S., Analyzing Politics. Rationality, Behavior and Institutions. Norton \& Company, Inc. New York, 1997. pp. 94

${ }^{8} \mathrm{El}$ win-set o conjunto ganador es vacio $\mathrm{W}(\mathrm{y})=Æ$ si no existe ninguna alternativa que pueda derrotar a y o bien $\mathrm{W}(\mathrm{y})^{1} Æ$ si existe alguna opción preferida a y.

${ }^{9}$ Shepsle, K. A. y Bonchek, M. S., op. cit., p. 96.
} 
Economía Informa núm. 389 noviembre - diciembre • 2014 | " " " " " " " " " " -

\section{Figura 3}

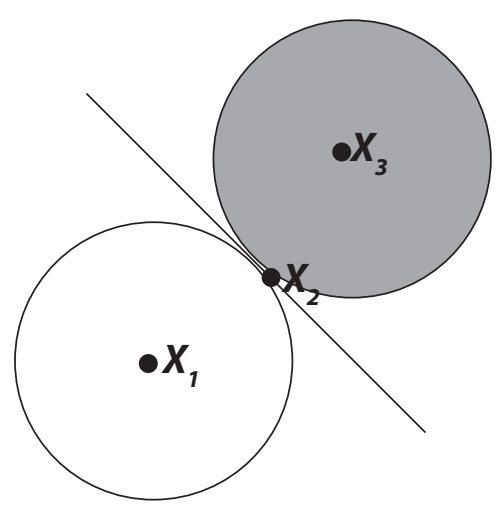

En la Figura 3 se establece la condición del votante mediano en un espacio bidimensional. En un esquema de tres votantes, cuyos puntos ideales satisface la simetría radial, $\mathrm{X}_{2}$ es la elección de mayoría. ${ }^{10}$ Este resultado es preferido por una mayoría a cualquier otro punto en el espacio de política. ${ }^{11}$ Es decir, si se garantiza que existen preferencias radiales y simétricas se puede garantizar la existencia de un ganador Condorcet multidimensional bajo la regla de la mayoría. Sin embargo, la condición de Plott de simetría radial es muy sensible a pequeñas perturbaciones en los puntos ideales de los votantes por lo que MacKelvey (1976) considera que es difícil encontrar situaciones de equilibrio.

\section{b) Teoría de los Jugadores de Veto (VP)}

Según Tsebélis los resultados políticos surgen de dos factores: las preferencias de los actores incluidos en el juego político y la existencia de instituciones. Si consideramos la variabilidad de la identidad de los jugadores y de sus preferencias pero mantenemos cierta estabilidad en las instituciones, entonces los resultados cambiaran y se modificaran dependiendo quien controle el poder político y donde se encuentre el Status Quo (SQ). Así, la estabilidad política de un sistema dependerá de la dificultad para efectuar un cambio significativo del sQ. (Tsebelis, 2002:17-21)

Uno de los elementos centrales que explican la estabilidad política son los jugadores de veto, que son actores individuales o colectivos cuyo acuerdo

\footnotetext{
${ }^{10}$ La generalización a más de una dimensión del teorema del votante mediano de Black se establece en el teorema de Plott que dice: "Si los votantes poseen preferencias espaciales basadas en la distancia, $y$ si sus puntos ideales están distribuidos de una manera simétrica y radial con $x *$ el principal punto ideal $y$ un número impar de votantes entonces $W(x *)=$ E"'.

${ }^{11}$ Shepsle, K. A. y Bonchek, M. S. , op. cit., pp. 99-100.
} 
es necesario para cambiar el SQ. Existen dos tipos de jugadores de veto: los institucionales y los partidarios. Si los jugadores de veto son generados por la constitución se llaman jugadores de veto institucionales pero, si surge del juego político (distribución de votos y escaños) ${ }^{12}$ serán jugadores partidarios.

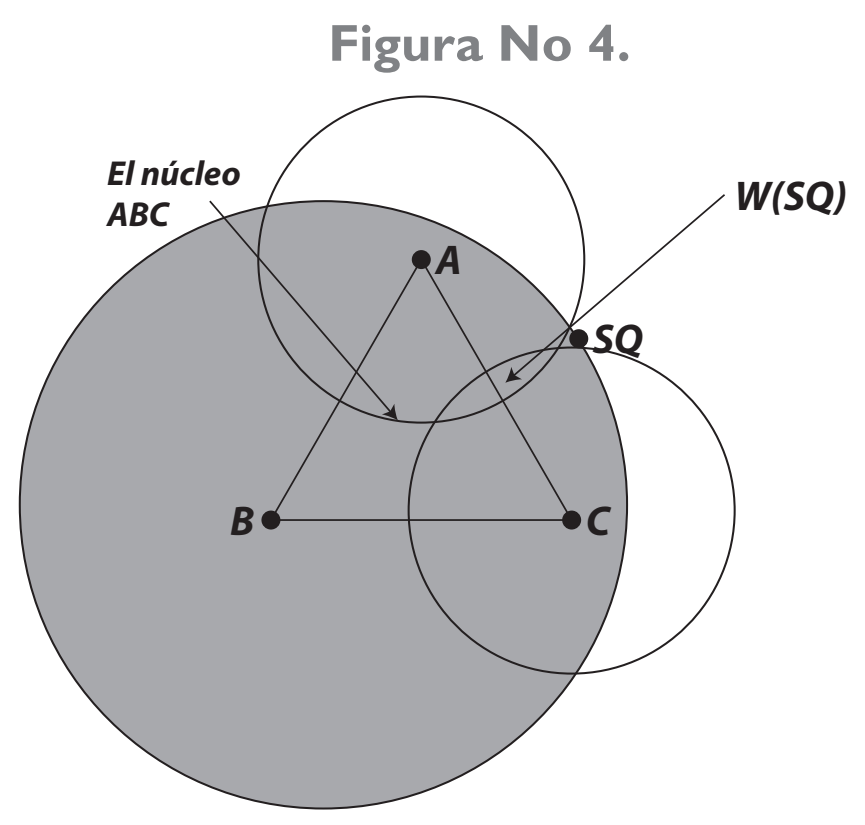

En la Figura 4, representamos dos conceptos importantes: conjunto ganador de SQ $(W(S Q))$, que es el conjunto de resultados que puede derrotar al SQ. $\mathrm{Y}$ el núcleo, que es el conjunto de puntos (triangulo $\mathrm{ABC}$ ) con un conjunto ganador vacío $(W(A B C)=Æ$ ), los puntos que no pueden ser derrotados por algún otro punto si aplicamos alguna regla para tomar decisiones. Por lo general nos referimos al núcleo en términos de la regla de decisión que lo origina, así el núcleo de unanimidad, es conocido como conjunto de pareto. (Tsebelis, 2002:21). En los sistemas políticos ciertos actores (agenda setter) hacen propuestas a otros quienes pueden aceptarlas o rechazarlas. Si consideramos tal secuencia de movimientos, podemos reducir significativamente la predicción de los posibles resultados. Por lo que es necesario no sólo saber la identidad del que pone la agenda sino conocer sus preferencias. De hecho, el VP que hace la propuesta considerará el conjunto ganador de todos los otros VP como su restricción, y seleccionará entre todos los puntos contenidos en el conjunto ganador uno que el prefiera. La importancia del agente que pone la

\footnotetext{
${ }^{12}$ Reynoso, D. (2004), Decisionismo y pluralismo en América Latina, Mimeo, p. 6.
} 
Economía Informa núm. 389 noviembre - diciembre • 2014 | " " " " " " " " " " -

agenda disminuye conforme se incrementa la estabilidad política. En el caso de jugadores de veto individuales es importante el número y la distancia para determinar la estabilidad del sistema político

Esto se podría modificar para el caso de jugadores de veto colectivos. Sin embargo, si asumimos que para modificar el SQ, se requiere del acuerdo de una cámara de la legislatura y que la cámara decide por mayoría simple de sus miembros, entonces la cámara es un jugador colectivo. Y ningún miembro individual de la legislatura tiene poder de veto sobre la legislación. Por lo que para encontrar el conjunto ganador del SQ tenemos que identificar las intersecciones de las curvas de indiferencia de todas las posibles mayorías. (Tsebelis, 2002:45).

\section{c) La elección pública tradicional y los grupos de presión}

Para la elección pública, los legisladores son agentes políticos racionales en un mercado legislativo. Es decir, en el poder legislativo existen demandantes y oferentes de legislación, que quieren una ley o una transferencia de recursos que afecte a muchos en detrimento de pocos (Rawley, 1993:xviii). Sin embargo, los políticos del Congreso, típicamente se centran en arreglos legales que benefician a grupos compactos y bien organizados, para quien el pro rateo de beneficios es alto, y que se cumple a expensa de grupos con intereses difusos (Tollison, 1988).

En general, los legisladores responden a grupos de presión o interés ${ }^{13}$ en este mercado político, en función de apoyos económicos, de votos o de posiciones laborales al finalizar el periodo legislativo (Rawley, 1993:xviii). Estos agentes hacen una evaluación costo beneficio presente e intertemporal en términos de los grupos beneficiados.

Para esta teoría, estos agentes políticos (brokers) son el corazón del proceso legislativo, buscan el equilibrio, con varios grados de error, reflejando las preferencias de los principales actores (económicos, políticos y sociales), condicionados por las reglas constitucionales y otras reglas que definen su mercado particular (Rawley, 1993:xix). Y como en todo mercado estos agentes políticos compiten por cumplir las demandas de determinados grupos de presión o bien se sujetan a una disciplina de partido que atienda a dichos grupos.

\footnotetext{
${ }^{13} \mathrm{Si}$ los ciudadanos o votantes al pensar que no pueden determinar los resultados electorales deciden permanecer racionalmente desinformados y no participar en las votaciones, este vació es ocupado por los grupos de presión. Rowley, C. K. (1993), Public Choice Theory, Edit. Galliard Ltd. Pág. xv.
} 
Un punto importante que subraya esta teoría es la importancia de los grupos parlamentarios, las comisiones y los comités como mecanismos que facilitan los acuerdos y disminuyen los costos de transacción del intercambio político. Esto podría facilitar la comprensión del intercambio de votos y el voto estratégico bastante utilizado en las legislaturas.

\section{Reforma Fiscal en la LVIII legislatura}

La alternancia en el Poder Ejecutivo en el año 2000 despertó grandes expectativas acerca de los cambios económicos, políticos y sociales que podía emprender la nueva administración. En el ámbito económico se planteaban grandes reformas estructurales acordes al Neo-Consenso de Washington de 1998: la reforma fiscal, la reforma energética y la reforma laboral, principalmente.

En cuanto a la reforma fiscal involucraba modificaciones sustanciales en el ingreso neto total del Gobierno, lo que se reflejaría en una modificación a la Ley de Ingresos y una redefinición del Presupuesto Anual de la Federación. ${ }^{14}$

Una reforma fiscal integral implicaba atender las diferentes fuentes de financiamiento o ingresos del gobierno. ${ }^{15}$ Sin embargo, esta propuesta se centro sólo en la modificación del Impuesto al Valor Agregado ${ }^{16}$ (IVA) sobre medicamentos, medicinas, libros y transporte. Inicialmente se planteaba pasar de una tasa cero a una tasa de $15 \%$, por considerar que este cambio incrementaría la eficiencia recaudatoria, incrementando los ingresos del Gobierno en 1.5 puntos porcentuales del PIB. En contrapartida, para proteger a las clases más vulnerables a este cambio, se incrementaría el gasto social a través de subsidios directos mediante Progresa. ${ }^{17}$

\footnotetext{
${ }^{14}$ En el caso de la Ley de ingresos, el proceso de discusión y aprobación involucra a los diputados y senadores. El Presidente envía su iniciativa a la Cámara de Diputados, ésta la examina y puede aprobarla o modificarla. Si la aprueba se turna al Senado y si éste la aprueba el Presidente tiene la obligación de promulgarla. Si es vetada por el Presidente, los legisladores deberán superar el veto con dos terceras partes de los votos. Mientras que el Presupuesto de Egresos es facultad exclusiva de la Cámara de Diputados. Para la reforma fiscal como la planteaba el Poder Ejecutivo se requería modificar una serie de leyes ordinarias: Ley de Impuestos sobre la Renta, Ley de Impuestos al Valor Agregado y Ley de Impuestos Especiales sobre la Producción.

${ }^{15}$ Ingresos tributarios y no tributarios, venta de bienes y servicios y endeudamiento interno y externo. Esto convierte la reforma en un asunto multidimensional. Sobre todo, al considerar que existe en algunos casos una Ley para cada impuesto y esta a su vez contiene diferentes artículos.

${ }^{16}$ En 1999, el IVA representaba $28.9 \%$ de los impuestos tributarios, el ISR $41.4 \%$ y otros impuestos aproximadamente el 29.7. Siendo los impuestos tributarios aproximadamente $46 \%$ de los ingresos totales.

${ }^{17}$ Se pensaba en una retribución de 110 pesos por familia a través de Progresa que beneficiaria a 4.9 millones de hogares y vía crédito fiscal a quienes ganaran menos de un salario mínimo.
} 
Economía Informa núm. 389 noviembre - diciembre • 2014 | " " " " " " " " " " -

Las modificaciones en la Ley de Ingreso y en las otras Leyes Ordinarias que permitiría la reforma implicaban la participación de tres jugadores de veto (vp) institucionales: El Presidente, la Cámara de Diputados y la Cámara de Senadores. ${ }^{18} \mathrm{Y}$ al interior de las Cámaras la aprobación de estas modificaciones requeriría de una mayoría simple $(1 / 2+1)$ por tratarse de una Ley Ordinaria, por lo que se pretendía de la participación de por lo menos dos jugadores de veto partidarios al interior de estas Cámaras, dependiendo que coalición permitiría alcanzar dicha mayoría. En el caso de la Cámara de Diputados el PRI tenía 207 escaños, el PAN 212 y el PRD 50. Y en la Cámara de Senadores el PRI 60, el PAN 46, el PRD 16 y el PVEM 5. ${ }^{19}$ Esta distribución de votos de los partidos más importantes nos permite establecer a los vP.

\section{Preferencias de los VP partidarios.}

En general, la posición y preferencias de dos de los vp institucionales surge del resultado de los ve partidarios de cada una de las Cámaras. Por lo que el análisis nos remite a establecer como se conformaron las preferencias y la ubicación de estos VP con relación a la reforma fiscal.

La fracción parlamentaria del PAN en la Cámara de Diputados y Senadores asumió la posición del Gobierno Federal de incrementar en $15 \%{ }^{20}$ el IVA en alimentos y medicinas. Aunque muchos legisladores estaban consientes del costo político y el impacto social, se alinearon a través de la disciplina partidista que se ejerce a través de los coordinadores parlamentarios. ${ }^{21}$ No obstante,

\footnotetext{
${ }^{18}$ La Cámara de Diputados y Senadores son un claro ejemplo de jugadores de veto colectivos, en este caso, en la Cámara de Diputados encontramos tres combinaciones: PRI-PAN, PRI-PRD y PAN-PRD y en el Senado: PRI-PAN, PRI-PRD y PRI-PVEM. De este modo se tenían 3 VP institucionales y 3 VP partidarios en cada Cámara, que dependían de las diferentes combinaciones para modificar el sQ. Reynoso, D. (2005). ¿Por qué el "gobierno del cambio” no cambió el Status Quo? Comentarios a la ponencia de Tsebelis sobre "Vetos y conflicto entre poderes en América Latina". Mimeo, pp. 12-14. ${ }^{19}$ Ibid, p. 30.

${ }^{20}$ Conforme transcurren las negociaciones y no se pudo establecer acuerdos con los otros partidos, el PAN modificó la propuesta inicial del Ejecutivo, pasando gradualmente a 12, 10, 6, 5 y 3\% en IVA en alimentos y medicinas. Hasta establecer un 5\% sobre el proceso de producción, industrialización, distribución y comercialización que fue rechazado por el PRI en las últimas horas de 29 de diciembre de 2001. Estas modificaciones en las preferencias del PAN podrían interpretarse como un acercamiento al núcleo de los otros vP.

${ }^{21}$ En México, la actividad o carrera política de los actores individuales depende de los partidos políticos. Estos definen las candidaturas o puestos burocráticos en los gobiernos que controlan. Así se tiene que si el legislador se alinea a las decisiones del partido, puede pasar de la Cámara de Diputados a la de Senadores o alguna Secretaria de Estado, etc.Y como los legisladores son agentes racionales que evalúan su relación costo beneficio en términos intertemporales, éstos se alinearan a las decisiones del partido pensando en su desarrollo político.
} 
para disminuir el impacto político plantearon respaldar un incremento en el Gasto Social.

Con relación a la posición y preferencias del Gobierno y de las elites del PAN que influyeron sobre los legisladores de este partido, encontramos que respondía a presiones presupuestarias en términos de deuda interna, externa y de pasivos contingentes ${ }^{22}$ que representaban $47 \%$ del PIB, y que podrían modificar algunas de las variables macroeconómicas ${ }^{23}$ como: la inflación, el tipo de cambio, las tasas de interés, la inversión, etc. causando un efecto negativo en la percepción de organismos internacionales y ciertos sectores empresariales nacionales ${ }^{24}$ e internacionales. Pero sobre todo, podría causar una parálisis del Gobierno por no tener los recursos suficientes para hacer frente a esta deuda.

El PRI después de perder la presidencia se ubico en el SQ como un votante mediano en los asuntos de un alto impacto político que afectaran a la población. El objetivo del PRI era crear una apreciación negativa del Gobierno del cambio en los sectores y bases electorales que había perdido en las elecciones anteriores. Esto operó para determinados grupos sociales pero, sobre todo para grupos de presión bien definidos. Es decir, el PRI intentó crear cierta inmovilidad en el Gobierno del presidente Fox pero sin afectar el curso de la economía. Si se creaba una inestabilidad económica por la indiferencia de este partido, se podría perder la aprobación de sectores de apoyo internacional y nacional. El PRI limitó los recursos públicos de la nueva administración al punto de que esta fuera solo funcional pero, que no tuviera un impacto político electoral..$^{25}$ No obstante benefició a los grupos empresariales al permitir la disminución del ISR de 40 a 32\%. Además de atender las demandas de la Asociación Nacional de Tiendas de Autoservicio y Departamentales (ANTAD) y de las diferentes asociaciones de farmacias ${ }^{26}$ que no estaban de acuerdo con estos cambios.

\footnotetext{
${ }^{22}$ La deuda reconocida por el sector público como proporción del PIB en el 2001 fue: Interna 10.7\%, externa 19\%; Pasivos Contingentes: IPAB 12\%, Piridengas 4\% y Bonos Carreteros 2\%. Total 47.1\%. El Financiero, 12 de febrero de 2001.

${ }^{23} \mathrm{Al}$ modificar los mecanismos de financiamiento y la percepción de los inversionistas nacionales y extranjeros que podrían originar una fuga de capitales.

${ }^{24}$ Es interesante analizar las posiciones de las diferentes cámaras empresariales: Concanaco, Concamin, Canacintra, Coparmex, CCE que se pronunciaban a favor de la reforma. Sobre todo, porque algunos de sus agremiados eran favorecidos por algunos rubros de la deuda: IPA, Piridengas o Bonos Carreteros. O por la estabilidad macroeconómica que daba certidumbre a sus inversiones. Aunque en realidad, algunos de estos grupos también serían beneficiado con las otras reformas económicas: la energética o la laboral. El Financiero. abril-junio de 2001.

${ }^{25} \mathrm{Y}$ en un sentido de maximización intertemporal bloquearía la reforma energética y laboral como carta de negociación con ciertos grupos de presión para las elecciones en el 2006 y 2012.

${ }^{26}$ La Asociación Nacional de Farmacias de México proponía 3\%, mientras que el Consejo Consul-
} 
Economía Informa núm. 389 noviembre - diciembre • 2014 | " " " " " " " " " " -

El PRI en función de sus preferencias aprovecho su experiencia legislativa para mantener el SQ en los temas que le convenían y modificar aquellos poco sustanciales o que le reportaran beneficios electorales o que beneficiaban a determinados grupos patrocinadores. ${ }^{27}$ En una primera etapa, el PRI atendiendo las recomendaciones de su Consejo Político Nacional y tratando de superar el golpe de la derrota federal del 2000, tuvo una gran cohesión interna y rechazo la reforma. Sin embargo, a partir de las primeras negociaciones y cabildeos del Gobierno Federal y del PAN para modificar el IVA, intentaron cooptar a ciertas facciones del PRI. ${ }^{28}$ Estos mecanismos no prosperaron por la cercanía de comicios electorales para Gobernador en Chiapas y Tabasco. El caso de Tabasco es significativo, por que la victoria en este estado definiría al nuevo presidente del partido.

En el caso del PRD sus preferencias se estructuraron en función de su electorado, este partido rechazaba totalmente la reforma fiscal por afectar a los grupos más vulnerables que forman parte de su base electoral. También tenía acercamiento con ciertos grupos empresariales que serían afectados por la reforma, por ejemplo la Unión Nacional de Propietarios de Farmacias de la República Mexicana, que son pequeños propietarios. Aun cuando sus preferencias estaban en bajar el IVA de 15 a 10\% en los artículos ya gravados, se acercaban completamente a la posición del PRI de mantener una tasa de $0 \%$ en alimentos y medicinas ${ }^{29}$. Este partido proponía otras alternativas como gravar las actividades financieras o modificar el IEPs que afectaban al tabaco, la gasolina y el alcohol.

tivo Farmacéutico de México y la Unión Nacional de Propietarios de Farmacias de la República Mexicana rechazaba cualquier incremento. El principal argumento era el impacto sobre los precios al consumidor lo que en conjunto con los alimentos ocasionaría un incremento de la inflación de 3 puntos porcentuales. Este incremento impactaría los salarios y la credibilidad en el Banco de México al no alcanzar $6.5 \%$ de inflación programada para ese año.

${ }^{27}$ A diferencia de otros países, en México después de la primera votación donde se decide modificar el SQ la Cámara de Diputados no vuelve a revisar la iniciativa completa. Las propuestas aprobadas en la primera votación se debaten y se modifica artículo por artículo, esto se conoce como sistema de votación prospectivo. Es decir, los legisladores votan modificar el SQ sin conocer cuál será el nuevo SQ. En Reynoso, D. (2004): Decisionismo y pluralismo en América Latina. Mimeo. Pág. 19. Este sistema fue aprovechado por el PRI para separar asuntos estratégicos, manteniendo o modificando aquellos que le beneficiaran.

${ }^{28}$ Ante la falta de la dirección central que había ejercido el presidente como jefe de partido, los gobernadores ocuparon ese vacío e intentaron negociar un incremento generalizado del IVA de 12\% con la atribución de poder recaudar 3\% a nivel local, propuesta que no avanzó.

${ }^{29}$ La propuesta de reforma del PRD consistía en incrementar el combate a la corrupción, reducir la evasión fiscal, revisar las exenciones de los sectores privilegiados, gravar transacciones especulativas, ampliar la base de los contribuyentes, etc. 


\section{Figura No 5.}

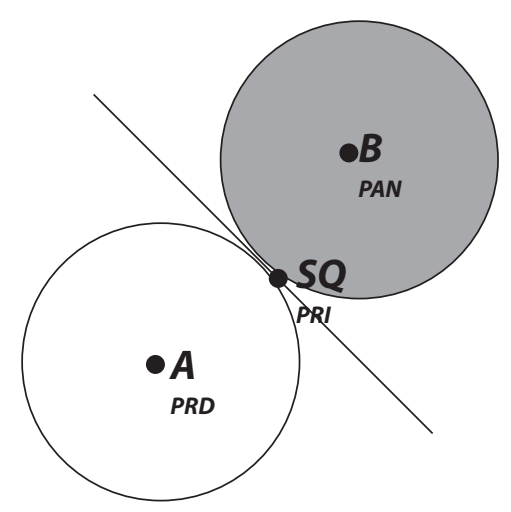

En la Figura No 5 el PRI se sitúa en $\mathrm{SQ}^{30}$ como votante mediano, esto implicaría que la reforma fiscal no procedería independientemente de la propuesta del PAN. Y como votante mediano estableció una coalición con el PRD para obtener la mayoría; la participación de los otros partidos no afectaría el resultado al tratarse de una mayoría de $(1 / 2+1) \cdot{ }^{31}$ En este caso, es la cantidad de diputados del PRI la que facilita su ubicación espacial para colocarse como un ganador Condorcet bidimensional, además de la ubicación del SQ y la distancia ideológica entre el PRD y el PAN, que difícilmente permitiría lograr una coalición que afectara el SQ.

El resultado final fue la derrota de la aplicación del IVA en alimentos y medicinas, que permitió que el SQ no se modificara. Sin embargo, se modificaron el ISR que pasó de 40 a 32\%, un impuesto de 20\% sobre bienes suntuarios, un gravamen de $5 \%$ sobre telecomunicaciones ${ }^{32}$ y algunas otras modificaciones.

\section{Comentarios finales}

Los cambios políticos, económicos y sociales que se esperaban de la alternancia del Poder Ejecutivo no fueron los esperados. El hecho de tener un gobierno dividido, en el que la mayoría legislativa pertenece a otro partido, implicó

\footnotetext{
${ }^{30}$ En general el PRI se sitúo como un votante mediano en casi todas las reformas estructurales por una lógica de maximización intertemporal, que le permitirá negociar su reubicación en las elecciones del 2006.

${ }^{31}$ La aplicación del IVA en alimentos y medicinas fue derrotada por el PRI, PRD, PVEM y PT.

${ }^{32}$ Se perdieron casi 4 mil millones de pesos al no gravar con $10 \%$ sino con $5 \%$ a las telecomunicaciones por propuesta del PRI. El Financiero, 2 de enero de 2002. Esto podría respaldar como los grupos de presión definen las preferencias de los legisladores.
} 
Economía Informa núm. 389 noviembre - diciembre • 2014 | " " " " " " " " " " -

que el PRI se ubicara en el SQ como votante mediano, no permitiendo que procedieran las reformas importantes para el nuevo gobierno.

La ubicación del SQ y las preferencias del grupo parlamentario del PRI podrían ser vistas en un contexto de elección intertemporal en el que se pretendía crear una parálisis en la nueva administración. En el caso del PRI como uno de los jugadores de veto más importantes sus preferencias intertemporales predominaron para no modificar el SQ.

Por otro lado, las decisiones de los jugadores de veto están sujetas no sólo a sus preferencias inmediatas sino a la rentabilidad y la disciplina partidista que afecta los beneficios y costos intertemporales de los partidos. $\mathrm{O}$ bien pueden ser formadas y modificadas por las preferencias de ciertos grupos de presión que tienen un alto peso en la vida económica del país.

\section{Bibliografía}

Aleman, Eduardo y George Tsebelis (2003), "Presidential Conditional Agenda Setting in Latin America", paper prepared for delivery at the Midwest Political Science Association, April 2-6.

Heller, William y Jeffrey Weldom (2003), "Reglas de Votación y la Estabilidad en la Cámara de Diputados”, en Luiza Bejar y Rosa María Mirón (coord): El Congreso Mexicano después de la Alternancia, AmeP, México

Hinich, Melvin y Michael Munger (1997), Analytical Politics, Cambridge University Press, Cambridge.

McKelvey, Richard (1976), "Intransitivity in Multidimensional Voting Models", Journal of Economic Theory 12, pp. 472-486.

Mueller, Denis (1997), Perspectives on Public Choice, A. Handbook. Cambrige University Press, U.S.A.

Plott, Charles (1967), “A Notion of Equilibrium and its Possibility under Majority Rule" American Economic Review 57, pp. 787-806.

Reynoso, Diego (2004), Decisionismo y pluralismo en América Latina, Mimeo. 34 p.

Reynoso, Diego (2005), ¿Por qué el "gobierno del cambio" no cambió el Status Quo? Comentarios a la ponencia de Tsebelis sobre "Vetos y conflicto entre poderes en América Latina, Mimeo, 34 p.

Rowley, C. K. (1993), Public Choice Theory (3 tomos). Edit. Galliard Ltd, Great Yarmouth. Gran Bretaña.

Shepsle, Kenneth y Mark Bonchek (1997), Analyzing Politics, Norton and Company, New York.

Tsebelis, George (2002): Veto Players: How Political Institutions Work, Russell Sage Fundation, Princeton. 